PROCEEDINGS OF THE

AMERICAN MATHEMATICAL SOCIETY

Volume 144, Number 4, April 2016, Pages 1717-1724

http://dx.doi.org/10.1090/proc/12850

Article electronically published on September 9, 2015

\title{
MODULI OF CONTINUITY FOR VISCOSITY SOLUTIONS
}

\author{
XIAOLONG LI
}

(Communicated by Michael Wolf)

\begin{abstract}
In this paper, we investigate the moduli of continuity for viscosity solutions of a wide class of nonsingular quasilinear evolution equations and also for the level set mean curvature flow, which is an example of singular degenerate equations. We prove that the modulus of continuity is a viscosity subsolution of some one-dimensional equation. This work extends B. Andrews' recent result on moduli of continuity for smooth spatially periodic solutions.
\end{abstract}

\section{INTRODUCTION}

Given a function $u: \mathbb{R}^{n} \rightarrow \mathbb{R}$, any function $f:[0, \infty) \rightarrow \mathbb{R}_{+}$satisfying

$$
|u(y)-u(x)| \leq 2 f\left(\frac{|y-x|}{2}\right)
$$

for all $x$ and $y$ is called a modulus of continuity of $u$. The (optimal) modulus of continuity $\omega$ of $u$ is defined by

$$
\omega(s)=\sup \left\{\frac{u(y)-u(x)}{2} \mid \frac{|y-x|}{2}=s\right\} .
$$

The estimate of modulus of continuity has been studied by B. Andrews and J. Clutterbuck in several papers [2] 3]. B. Andrews and J. Clutterbuck [4, B. Andrews and L. Ni [5] and L. Ni [8] have also studied the modulus of continuity for heat equations on manifolds.

More precisely, B. Andrews and J. Clutterbuck considered the following quasilinear evolution equation

$$
u_{t}=a^{i j}(D u, t) D_{i} D_{j} u+b(D u, t),
$$

where $A(p, t)=\left(a^{i j}(p, t)\right)$ is positive semidefinite. Under the assumption that there exists a continuous function $\alpha: \mathbb{R}_{+} \times[0, T] \rightarrow \mathbb{R}$ with

$$
0<\alpha(R, t) \leq R^{2} \inf _{|p|=R,(v \cdot p) \neq 0} \frac{v^{T} A(p, t) v}{(v \cdot p)^{2}},
$$

they showed [3, Theorem 3.1] that the modulus of continuity of a regular periodic solution to (1.1) is a viscosity subsolution of the one-dimensional equation

$$
\phi_{t}=\alpha\left(\left|\phi^{\prime}\right|, t\right) \phi^{\prime \prime} .
$$

Note that their result is applicable to any anisotropic mean curvature flow and can be used to obtain gradient estimate and thus existence and uniqueness of (1.1).

Received by the editors April 17, 2015.

2010 Mathematics Subject Classification. Primary 53C44; Secondary 35D40.

Key words and phrases. Modulus of continuity, viscosity solution, level set mean curvature flow. 
The first result of this paper is that the same holds for viscosity solutions of (1.1) when (1.2) holds and $a^{i j}, b: \mathbb{R}^{n} \times[0, T] \rightarrow \mathbb{R}$ are continuous functions.

Theorem 1.1. Let $u: \mathbb{R}^{n} \times[0, T] \rightarrow \mathbb{R}$ be a continuous periodic viscosity solution of (1.1). Then the modulus of continuity $\omega(s, t)=\sup \left\{\frac{u(y, t)-u(x, t)}{2} \mid \frac{|y-x|}{2}=s\right\}$ of $u$ is a viscosity subsolution of the one-dimensional equation (1.3).

We also study the modulus of continuity for singular evolution equations. As summarized in a recent survey [1] by B. Andrews, for the isotropic flows of the form

$$
u_{t}=\left[a(|D u|) \frac{D_{i} u D_{j} u}{|D u|^{2}}+b(|D u|)\left(\delta_{i j}-\frac{D_{i} u D_{j} u}{|D u|^{2}}\right)\right] D_{i} D_{j} u,
$$

the modulus of continuity of a spatially periodic smooth solution of (1.4) is a viscosity subsolution of the corresponding one-dimensional heat equation $\omega_{t}=a\left(\omega^{\prime}\right) \omega^{\prime \prime}$. Note that equation (1.4) covers the classical heat equation, the graphical mean curvature flow and the $p$-Laplace heat equation with suitable choices of $a$ and $b$. When (1.4) is nonsingular, it is covered by (1.1). When it is singular, it has to be treated differently since there are various definitions for viscosity solutions of singular equations. We will focus on the particular case $a=0$ and $b=1$, which corresponds to the level set mean curvature flow:

$$
u_{t}=\left(\delta_{i j}-\frac{D_{i} u D_{j} u}{|D u|^{2}}\right) D_{i} D_{j} u .
$$

Equation (1.5) was studied by L. Evans and J. Spruck in 7]. They gave a definition of viscosity solution and proved that for an initial data $g$ that is continuous and constant on $\mathbb{R}^{n} \cap\{|x| \geq S\}$, there exists a unique viscosity solution $u$ that is continuous and constant on $\mathbb{R}^{n} \cap\{|x| \geq R\}$, with $R$ depending only on $S$. We will recall their definition in Section 2.

We prove the following theorem:

Theorem 1.2. Let $u: \mathbb{R}^{n} \times[0, \infty) \rightarrow \mathbb{R}$ be a viscosity solution of (1.5) with continuous initial data $g$ that is a constant on $\mathbb{R}^{n} \cap\{|x| \geq S\}$. Then the modulus of continuity $\omega(s, t)=\sup \left\{\frac{u(y, t)-u(x, t)}{2} \mid \frac{|y-x|}{2}=s\right\}$ of $u$ is a viscosity subsolution of $\omega_{t}=\max \left\{0, \frac{1}{4}\left(\omega^{\prime \prime}+\left|\omega^{\prime \prime}\right|\right)\right\}$ on $(0, \infty) \times(0, \infty)$.

As an immediate consequence, we have that any concave modulus of continuity for the initial data is preserved by the level set mean curvature flow.

Corollary 1.3. Let $u: \mathbb{R}^{n} \times[0, \infty) \rightarrow \mathbb{R}$ be a viscosity solution of (1.5) with continuous initial data $g$ that is a constant on $\mathbb{R}^{n} \cap\{|x| \geq S\}$. Assume $\phi$ is nonnegative, concave and satisfies $|g(y)-g(x)| \leq 2 \phi\left(\frac{|y-x|}{2}\right)$ for all $x, y$. Then

$$
|u(y, t)-u(x, t)| \leq 2 \phi\left(\frac{|y-x|}{2}\right)
$$

for all $x, y$ and $t \geq 0$.

Proof of Corollary 1.3. Since the function $\phi_{\epsilon}=\phi+\epsilon e^{t}$ satisfies

$$
\partial_{t} \phi_{\epsilon}>0=\max \left\{0, \frac{1}{4}\left(\phi^{\prime \prime}+\left|\phi^{\prime \prime}\right|\right)\right\},
$$

it cannot touch $\omega$ from above by Theorem 1.2 


\section{Definitions of Viscosity SOlutions}

We give a definition of a viscosity solution for the general equation

$$
u_{t}+F\left(x, t, u, \nabla u, \nabla^{2} u\right)=0,
$$

assuming $F: \mathbb{R}^{n} \times[0, T] \times \mathbb{R} \times \mathbb{R}^{n} \times S^{n \times n} \rightarrow \mathbb{R}$ is continuous and degenerate elliptic.

Let $O$ be an open subset of $\Omega \times(0, T)$. We write $z=(x, t)$ and $z_{0}=\left(x_{0}, t_{0}\right)$.

The following notations are useful:

$$
\begin{aligned}
& \operatorname{USC}(O)=\{u: O \rightarrow \mathbb{R} \mid u \text { is upper semicontinuous }\}, \\
& \operatorname{LSC}(O)=\{u: O \rightarrow \mathbb{R} \mid u \text { is lower semicontinuous }\} .
\end{aligned}
$$

Definition 2.1. (i) A function $u \in \operatorname{USC}(O)$ is a viscosity subsolution of (2.1) in $O$ if for any $\phi \in C^{\infty}(O)$ such that $u-\phi$ has a local maximum at $z_{0} \in O$, then

$$
\phi_{t}\left(z_{0}\right)+F\left(z_{0}, u\left(z_{0}\right), \nabla \phi\left(z_{0}\right), \nabla^{2} \phi\left(z_{0}\right)\right) \leq 0 .
$$

(ii) A function $u \in \operatorname{LSC}(O)$ is a viscosity supersolution of (2.1) in $O$ if for any $\phi \in C^{\infty}(O)$ such that $u-\phi$ has a local minimum at $z_{0} \in O$, then

$$
\phi_{t}\left(z_{0}\right)+F\left(z_{0}, u\left(z_{0}\right), \nabla \phi\left(z_{0}\right), \nabla^{2} \phi\left(z_{0}\right)\right) \geq 0 .
$$

(iii) A viscosity solution of (2.1) in $O$ is defined to be a continuous function that is both a viscosity subsolution and a viscosity supersolution of (2.1) in $O$.

We have an equivalent definition in terms of parabolic semijets. Assume $u \in$ $\operatorname{USC}(O)$ and $z_{0} \in O$. The parabolic superjet of $u$ at $z_{0}$, denoted by $\mathcal{P}^{2,+} u\left(z_{0}\right)$, is defined by

$$
\begin{aligned}
\mathcal{P}^{2,+} u\left(z_{0}\right)= & \left\{(\tau, p, X) \in \mathbb{R} \times \mathbb{R}^{n} \times S^{n \times n} \mid u(z) \leq u\left(z_{0}\right)+\tau\left(t-t_{0}\right)+p \cdot\left(x-x_{0}\right)\right. \\
& \left.+\frac{1}{2}\left(x-x_{0}\right)^{T} X\left(x-x_{0}\right)+o\left(\left|x-x_{0}\right|^{2}+\left|t-t_{0}\right|\right) \text { as } z \rightarrow z_{0}\right\} .
\end{aligned}
$$

The parabolic subjet of $u \in \operatorname{LSC}(O)$ at $z_{0}$, denoted by $\mathcal{P}^{2,-} u\left(z_{0}\right)$, is defined by

$$
\mathcal{P}^{2,-} u\left(z_{0}\right)=-\mathcal{P}^{2,+}(-u)\left(z_{0}\right) \text {. }
$$

Definition 2.2. (i) A function $u \in \operatorname{USC}(O)$ is a viscosity subsolution of (2.1) in $O$ if for all $(x, t) \in O$ and $(\tau, p, X) \in \mathcal{P}^{2,+} u(x, t)$,

$$
\tau+F(z, u(z), p, X) \leq 0
$$

(ii) A function $u \in \operatorname{LSC}(O)$ is a viscosity supersolution of (2.1) in $O$ if for all $(x, t) \in O$ and $(\tau, p, X) \in \mathcal{P}^{2,-} u(x, t)$,

$$
\tau+F(z, u(z), p, X) \geq 0 .
$$

Remark 2.3. In the above definitions, since $F$ is continuous, we can replace $\mathcal{P}^{2,+} u\left(z_{0}\right)$ and $\mathcal{P}^{2,-} u\left(z_{0}\right)$ by $\overline{\mathcal{P}}^{2,+} u\left(z_{0}\right)$ and $\overline{\mathcal{P}}^{2,-} u\left(z_{0}\right)$ respectively, where the closures are defined by

$$
\begin{aligned}
& \overline{\mathcal{P}}^{2,+} u\left(z_{0}\right)=\{((\tau, p, X) \in \mathbb{R} \times \mathbb{R}^{n} \times S^{n \times n} \mid \text { there is a sequence }\left(z_{j}, \tau_{j}, p_{j}, X_{j}\right) \\
& \text { such that }\left(\tau_{j}, p_{j}, X_{j}\right) \in \mathcal{P}^{2,+} u\left(z_{j}\right) \\
&\text { and } \left.\left(z_{j}, u\left(z_{j}\right), \tau_{j}, p_{j}, X_{j}\right) \rightarrow\left(z_{0}, u\left(z_{0}\right) \tau, p, X\right) \text { as } j \rightarrow \infty\right\} . \\
& \overline{\mathcal{P}}^{2,-} u\left(z_{0}\right)=-\overline{\mathcal{P}}^{2,+}(-u)\left(z_{0}\right) .
\end{aligned}
$$


For singular equations, there are different ways to define viscosity solutions. For the level set mean curvature flow (1.5), we use the definition given by L. Evans and J. Spruck in [7, where viscosity solutions are called weak solutions.

Definition 2.4. A continuous and bounded function $u: \mathbb{R}^{n} \times[0, \infty) \rightarrow \mathbb{R}$ is a viscosity subsolution of (1.5) if for any $\phi \in C^{\infty}\left(\mathbb{R}^{n+1}\right)$ such that $u-\phi$ has a local maximum at a point $\left(x_{0}, t_{0}\right) \in \mathbb{R}^{n} \times(0, \infty)$, then we have

$$
\left\{\begin{array}{l}
\phi_{t} \leq\left(\delta_{i j}-\frac{D_{i} \phi D_{j} \phi}{|D \phi|^{2}}\right) D_{i} D_{j} \phi \text { at }\left(x_{0}, t_{0}\right) \\
\text { if } D \phi\left(x_{0}, t_{0}\right) \neq 0,
\end{array}\right.
$$

and

$$
\left\{\begin{array}{l}
\phi_{t} \leq\left(\delta_{i j}-\eta_{i} \eta_{j}\right) D_{i} D_{j} \phi \text { at }\left(x_{0}, t_{0}\right) \\
\text { for some } \eta \in \mathbb{R}^{n} \text { with }|\eta| \leq 1, \text { if } D \phi\left(x_{0}, t_{0}\right)=0 .
\end{array}\right.
$$

Definition 2.5. A continuous and bounded function $u: \mathbb{R}^{n} \times[0, \infty) \rightarrow \mathbb{R}$ is a viscosity supersolution of (1.5) if for any $\phi \in C^{\infty}\left(\mathbb{R}^{n+1}\right)$ such that $u-\phi$ has a local minimum at a point $\left(x_{0}, t_{0}\right) \in \mathbb{R}^{n} \times(0, \infty)$, then we have

$$
\left\{\begin{array}{l}
\phi_{t} \geq\left(\delta_{i j}-\frac{D_{i} \phi D_{j} \phi}{|D \phi|^{2}}\right) D_{i} D_{j} \phi \text { at }\left(x_{0}, t_{0}\right) \\
\text { if } D \phi\left(x_{0}, t_{0}\right) \neq 0,
\end{array}\right.
$$

and

$$
\left\{\begin{array}{l}
\phi_{t} \geq\left(\delta_{i j}-\eta_{i} \eta_{j}\right) D_{i} D_{j} \phi \text { at }\left(x_{0}, t_{0}\right) \\
\text { for some } \eta \in \mathbb{R}^{n} \text { with }|\eta| \leq 1, \text { if } D \phi\left(x_{0}, t_{0}\right)=0 .
\end{array}\right.
$$

Definition 2.6. A continuous and bounded function $u: \mathbb{R}^{n} \times[0, \infty) \rightarrow \mathbb{R}$ is a viscosity solution of (1.5) provided $u$ is both a viscosity subsolution and a viscosity supersolution.

We also have alternative definitions in terms of parabolic semijets.

Definition 2.7. A continuous and bounded function $u: \mathbb{R}^{n} \times[0, \infty) \rightarrow \mathbb{R}$ is a viscosity subsolution of (1.5) if for all $(x, t) \in \mathbb{R}^{n} \times(0, \infty)$ and $(\tau, p, X) \in \mathcal{P}^{2,+} u(x, t)$,

$$
\tau \leq\left(\delta_{i j}-\frac{p_{i} p_{j}}{|p|^{2}}\right) X_{i j} \text { if } p \neq 0
$$

and

$$
\tau \leq\left(\delta_{i j}-\eta_{i} \eta_{j}\right) X_{i j} \text { for some }|\eta| \leq 1 \text {, if } p=0 .
$$

Definition 2.8. A continuous and bounded function $u: \mathbb{R}^{n} \times[0, \infty) \rightarrow \mathbb{R}$ is a viscosity supersolution of (1.5) if for all $(x, t) \in \mathbb{R}^{n} \times(0, \infty)$ and $(\tau, p, X) \in$ $\mathcal{P}^{2,-} u(x, t)$

$$
\tau \geq\left(\delta_{i j}-\frac{p_{i} p_{j}}{|p|^{2}}\right) X_{i j} \text { if } p \neq 0
$$

and

$$
\tau \geq\left(\delta_{i j}-\eta_{i} \eta_{j}\right) X_{i j} \text { for some }|\eta| \leq 1 \text {, if } p=0 .
$$

Remark 2.9. One can replace $\mathcal{P}^{2,+} u\left(z_{0}\right)$ and $\mathcal{P}^{2,-} u\left(z_{0}\right)$ by $\overline{\mathcal{P}}^{2,+} u\left(z_{0}\right)$ and $\overline{\mathcal{P}}^{2,-} u\left(z_{0}\right)$ respectively in the above definitions for the reason of continuity. 


\section{Proof of Theorem 1.1}

Proof of Theorem 1.1. We must show that if $\phi$ is a smooth function such that $\omega-\phi$ has a local maximum at $\left(s_{0}, t_{0}\right)$ for $s_{0}>0$ and $t_{0}>0$, then at $\left(s_{0}, t_{0}\right)$

$$
\phi_{t} \leq \alpha\left(\left|\phi^{\prime}\right|, t\right) \phi^{\prime \prime} .
$$

Since $u$ is continuous and periodic, there exist points $x_{0}$ and $y_{0}$ with $\left|y_{0}-x_{0}\right|=2 s_{0}$ attaining the supremum,

$$
\omega\left(s_{0}, t_{0}\right)=\frac{u\left(y_{0}, t_{0}\right)-u\left(x_{0}, t_{0}\right)}{2} .
$$

Define

$$
Z(x, y, t):=u(y, t)-u(x, t)-2 \phi\left(\frac{|y-x|}{2}, t\right) .
$$

In view of the definition of $\omega$, we obtain that

$$
Z(x, y, t) \leq Z\left(x_{0}, y_{0}, t_{0}\right)
$$

for all $|y-x|$ close to $2 s_{0}$ and $t$ close to $t_{0}$. Thus $Z$ has a local maximum at $\left(x_{0}, y_{0}, t_{0}\right)$. Since $Z$ is continuous, by the parabolic version maximum principle for semicontinuous functions [6. Theorem 8.3], for any $\lambda>0$, there exist $X, Y \in S^{n \times n}$ such that

$$
\begin{gathered}
\left(b_{1}, 2 D_{y} \phi\left(s_{0}, t_{0}\right), X\right) \in \overline{\mathcal{P}}^{2,+} u\left(y_{0}, t_{0}\right), \\
\left(-b_{2},-2 D_{x} \phi\left(s_{0}, t_{0}\right), Y\right) \in \overline{\mathcal{P}}^{2,-} u\left(x_{0}, t_{0}\right), \\
b_{1}+b_{2}=2 \phi_{t}\left(s_{0}, t_{0}\right), \\
-\left(\lambda^{-1}+\|M\|\right) I \leq\left(\begin{array}{cc}
X & 0 \\
0 & -Y
\end{array}\right) \leq M+\lambda M^{2},
\end{gathered}
$$

where

$$
M=2 D^{2} \phi=2\left(\begin{array}{cc}
D_{y}^{2} \phi & D_{y, x}^{2} \phi \\
D_{x, y}^{2} \phi & D_{x}^{2} \phi
\end{array}\right)=\left(\begin{array}{cc}
B & -B \\
-B & B
\end{array}\right)
$$

with $B=2 D_{y}^{2} \phi\left(s_{0}, t_{0}\right)$.

To simplify, we choose an orthonormal basis of $\mathbb{R}^{n}$ with $e_{n}=\frac{y-x}{|y-x|}$, then

$$
\begin{gathered}
2 D_{y} \phi\left(s_{0}, t_{0}\right)=-2 D_{x} \phi\left(s_{0}, t_{0}\right)=\phi^{\prime}\left(s_{0}, t_{0}\right) e_{n}, \\
B=\left(\begin{array}{cccc}
\frac{\phi^{\prime}}{2 s_{0}} & & & \\
& \ddots & & \\
& & \frac{\phi^{\prime}}{2 s_{0}} & \\
& & & \frac{1}{2} \phi^{\prime \prime}
\end{array}\right) .
\end{gathered}
$$

Since $u$ is both a subsolution and a supersolution of (1.1), we have

$$
\begin{gathered}
b_{1} \leq \operatorname{tr}\left(A\left(\phi^{\prime} e_{n}\right) X\right)+b\left(\phi^{\prime} e_{n}\right), \\
-b_{2} \geq \operatorname{tr}\left(A\left(\phi^{\prime} e_{n}\right) Y\right)+b\left(\phi^{\prime} e_{n}\right) .
\end{gathered}
$$


By choosing a symmetric matrix $C$ such that $\left(\begin{array}{ll}A & C \\ C & A\end{array}\right) \geq 0$, we obtain using (3.1)

$$
\begin{aligned}
2 \phi_{t}\left(s_{0}, t_{0}\right) & =b_{1}+b_{2} \leq \operatorname{tr}\left(A\left(\phi^{\prime} e_{n}\right)(X-Y)\right)=\operatorname{tr}\left(\begin{array}{ll}
A & C \\
C & A
\end{array}\right)\left(\begin{array}{cc}
X & 0 \\
0 & -Y
\end{array}\right) \\
& \leq \operatorname{tr}\left(\begin{array}{ll}
A & C \\
C & A
\end{array}\right)\left(\begin{array}{cc}
B & -B \\
-B & B
\end{array}\right)+\lambda \operatorname{tr}\left(\begin{array}{cc}
A & C \\
C^{T} & A
\end{array}\right)\left(\begin{array}{cc}
B & -B \\
-B & B
\end{array}\right)^{2} \\
& =2 \operatorname{tr}((A-C) B)+4 \lambda \operatorname{tr}\left((A-C) B^{2}\right) .
\end{aligned}
$$

Taking $C=A-2 \alpha\left(\left|\phi^{\prime}\right|\right) e_{n} \otimes e_{n}$, it's easy to verify $\left(\begin{array}{ll}A & C \\ C & A\end{array}\right) \geq 0$ due to (1.2). Thus at $\left(s_{0}, t_{0}\right)$

$$
\phi_{t} \leq \alpha\left(\left|\phi^{\prime}\right|\right) \phi^{\prime \prime}+\lambda \alpha\left(\left|\phi^{\prime}\right|\right)\left(\phi^{\prime \prime}\right)^{2} .
$$

Since $\lambda>0$ is arbitrary, we get

$$
\phi_{t} \leq \alpha\left(\left|\phi^{\prime}\right|\right) \phi^{\prime \prime}
$$

at $\left(s_{0}, t_{0}\right)$.

\section{Proof of Theorem 1.2}

Proof of Theorem 1.2. Suppose that $\phi$ is a smooth function such that $\omega-\phi$ has a strict local maximum at $\left(s_{0}, t_{0}\right)$ with $s_{0}>0$ and $t_{0}>0$. As in the proof of Theorem 1.1. we conclude that the function

$$
Z(x, y, t):=u(y, t)-u(x, t)-2 \phi\left(\frac{|y-x|}{2}, t\right)
$$

has a local maximum at $\left(x_{0}, y_{0}, t_{0}\right)$. Again we use an orthonormal frame with $e_{n}=\frac{y-x}{|y-x|}$. The maximum principle for semicontinuous functions [6, Theorem 8.3] implies that for any $\lambda>0$, there exist $X, Y \in S^{n \times n}$ such that

$$
\begin{gathered}
\left(b_{1}, \phi^{\prime}\left(s_{0}, t_{0}\right) e_{n}, X\right) \in \overline{\mathcal{P}}^{2,+} u\left(y_{0}, t_{0}\right), \\
\left(-b_{2}, \phi^{\prime}\left(s_{0}, t_{0}\right) e_{n}, Y\right) \in \overline{\mathcal{P}}^{2,-} u\left(x_{0}, t_{0}\right), \\
b_{1}+b_{2}=2 \phi_{t}\left(s_{0}, t_{0}\right), \\
-\left(\lambda^{-1}+\|M\|\right) I \leq\left(\begin{array}{cc}
X & 0 \\
0 & -Y
\end{array}\right) \leq M+\lambda M^{2},
\end{gathered}
$$

where

$$
M=\left(\begin{array}{cc}
B & -B \\
-B & B
\end{array}\right)
$$

with

$$
B=2 D_{y}^{2} \phi\left(s_{0}, t_{0}\right)=\left(\begin{array}{cccc}
\frac{\phi^{\prime}}{2 s_{0}} & & & \\
& \ddots & & \\
& & \frac{\phi^{\prime}}{2 s_{0}} & \\
& & & \frac{1}{2} \phi^{\prime \prime}
\end{array}\right) .
$$

For any vector $p \in \mathbb{R}^{n}$, we have

$$
p^{T} X p-p^{T} Y p=(p, p)^{T}\left(\begin{array}{cc}
X & 0 \\
0 & -Y
\end{array}\right)(p, p) \leq(p, p)^{T}\left(M+\lambda M^{2}\right)(p, p)=0 .
$$


Therefore $X \leq Y$. For simplicity, we denote $A(p)=I-\frac{p \otimes p}{|p|^{2}}$. If $\phi^{\prime}\left(s_{0}, t_{0}\right) \neq 0$, then by the definition of viscosity solution of (1.5),

$$
\begin{aligned}
b_{1} & \leq \operatorname{tr}\left(A\left(\phi^{\prime} e_{n}\right) X\right), \\
-b_{2} & \geq \operatorname{tr}\left(A\left(\phi^{\prime} e_{n}\right) Y\right) .
\end{aligned}
$$

Using the fact $X \leq Y$, we get

$$
\phi_{t}=\frac{1}{2}\left(b_{1}+b_{2}\right) \leq \frac{1}{2} \operatorname{tr}\left(A\left(\phi^{\prime} e_{n}\right)(X-Y)\right) \leq 0 .
$$

If $\phi^{\prime}\left(s_{0}, t_{0}\right)=0$, then it follows from the definition of a viscosity solution that for some $\xi, \eta$ with $|\xi|,|\eta| \leq 1$,

$$
\begin{aligned}
b_{1} & \leq \operatorname{tr}(A(\xi) X), \\
-b_{2} & \geq \operatorname{tr}(A(\eta) Y) .
\end{aligned}
$$

In view of (4.1), we have $X \leq B+2 \lambda B^{2}$ and $-Y \leq B+2 \lambda B^{2}$. Thus

$$
\begin{gathered}
\operatorname{tr}((A(\xi) X)) \leq \operatorname{tr}\left(\left(A(\xi)\left(B+2 \lambda B^{2}\right)\right)=\frac{1}{2}\left(1-\xi_{n}^{2}\right)\left(\left(\phi^{\prime \prime}+\lambda\left(\phi^{\prime \prime}\right)^{2}\right),\right.\right. \\
\operatorname{tr}((A(\eta) Y)) \geq \operatorname{tr}\left(\left(A(\eta)\left(-B-2 \lambda B^{2}\right)\right)=-\frac{1}{2}\left(1-\eta_{n}^{2}\right)\left(\left(\phi^{\prime \prime}+\lambda\left(\phi^{\prime \prime}\right)^{2}\right) .\right.\right.
\end{gathered}
$$

Therefore,

$$
2 \phi_{t}=b_{1}+b_{2} \leq \operatorname{tr}(A(\xi) X)-\operatorname{tr}(A(\eta) Y) \leq\left(1-\frac{\xi_{n}^{2}+\eta_{n}^{2}}{2}\right)\left(\phi^{\prime \prime}+\lambda\left(\phi^{\prime \prime}\right)^{2}\right) .
$$

Letting $\lambda \rightarrow 0$ yields

$$
\phi_{t} \leq \frac{1}{4}\left(\phi^{\prime \prime}+\left|\phi^{\prime \prime}\right|\right)
$$

\section{ACKNOWLEDGEMENT}

The author would like to thank his advisors Professor Lei Ni and Professor Ben Chow for their guidance and encouragement.

\section{REFERENCES}

[1] Ben Andrews, Moduli of continuity, isoperimetric profiles, and multi-point estimates in geometric heat equations, preprint.

[2] Ben Andrews and Julie Clutterbuck, Lipschitz bounds for solutions of quasilinear parabolic equations in one space variable, J. Differential Equations 246 (2009), no. 11, 4268-4283, DOI 10.1016/j.jde.2009.01.024. MR2517770 (2010j:35237)

[3] Ben Andrews and Julie Clutterbuck, Time-interior gradient estimates for quasilinear parabolic equations, Indiana Univ. Math. J. 58 (2009), no. 1, 351-380, DOI 10.1512/iumj.2009.58.3756. MR.2504416 (2010k:35234)

[4] Ben Andrews and Julie Clutterbuck, Sharp modulus of continuity for parabolic equations on manifolds and lower bounds for the first eigenvalue, Anal. PDE 6 (2013), no. 5, 1013-1024, DOI 10.2140/apde.2013.6.1013. MR 3125548

[5] Ben Andrews and Lei Ni, Eigenvalue comparison on Bakry-Emery manifolds, Comm. Partial Differential Equations 37 (2012), no. 11, 2081-2092, DOI 10.1080/03605302.2012.668602. MR3005536

[6] Michael G. Crandall, Hitoshi Ishii, and Pierre-Louis Lions, User's guide to viscosity solutions of second order partial differential equations, Bull. Amer. Math. Soc. (N.S.) 27 (1992), no. 1, 1-67, DOI 10.1090/S0273-0979-1992-00266-5. MR1118699 (92j:35050)

[7] L. C. Evans and J. Spruck, Motion of level sets by mean curvature. I, J. Differential Geom. 33 (1991), no. 3, 635-681. MR1100206 (92h:35097) 
[8] Lei $\mathrm{Ni}$, Estimates on the modulus of expansion for vector fields solving nonlinear equations (English, with English and French summaries), J. Math. Pures Appl. (9) 99 (2013), no. 1, 1-16, DOI 10.1016/j.matpur.2012.05.009. MR.3003280

Department of Mathematics, University of California, San Diego, La Jolla, CaliFORNIA 92093

E-mail address: xil117@ucsd.edu 